\title{
Explicit formula of a new class of $q$-Hermite-based Apostol-type polynomials and generalization
}

\author{
Mouloud Goubi \\ Department of Mathematics, University of UMMTO \\ Tizi-Ouzou 15000, Algeria \\ Algebra and Number Theory Laboratory, USTHB \\ Algiers, Algeria \\ e-mail: mouloud.goubi@ummto.dz
}

Received: 4 June 2020

Revised: 31 October 2020

Accepted: 4 November 2020

\begin{abstract}
The present article deals with a recent study of a new class of $q$-Hermite-based Apostol-type polynomials introduced by Waseem A. Khan and Divesh Srivastava. We give their explicit formula and study a generalized class depending in any $q$ - analog generating function.
\end{abstract}

Keywords: $q$-Hermite-based Apostol-type polynomials, $q$-analog Cauchy product, $f_{q}$-Hermitebased Apostol-type polynomials and numbers.

2010 Mathematics Subject Classification: 05A10, 05A15, 11B68, 16 B65.

\section{Introduction}

Throughout this work, $\mathbb{C}$ designates the field of complex numbers, $\mathbb{N}$ indicates the set of positive integers and $\mathbb{N}^{\star}=\mathbb{N} \backslash\{0\}$. First we recall some concepts related to $q$-calculus, which we need in the development of this article. Let $(a, q) \in \mathbb{C}$ such that $|q|<1$. The $q$-analog of $a$ is given by

$$
[a]_{q}=\frac{1-q^{a}}{1-q}
$$

and the $q$-factorial function is defined by

$$
[n]_{q} !=\prod_{m=0}^{n}[m]_{q}=\frac{(q ; q)_{n}}{(1-q)^{n}}
$$


with $(q ; q)_{n}=\prod_{m=1}^{n}\left(1-m^{q}\right)$. The corresponding $q$-binomial coefficient is given by the relation

$$
\left(\begin{array}{l}
n \\
k
\end{array}\right)_{q}=\frac{[n]_{q} !}{[k]_{q} ![n-k]_{q} !}=\frac{(q ; q)_{n}}{(q ; q)_{k}(q ; q)_{n-k}} .
$$

Finally the $q$-exponential generating function is defined by

$$
e_{q}(t)=\sum_{n \geq 0} \frac{t^{n}}{[n]_{q} !}=\sum_{n \geq 0} \frac{(1-q)^{n}}{(q ; q)_{n}} t^{n} .
$$

According to these notations, the $q$-Hermite polynomials $H_{n, q}(x)$ are defined by means of the generating function (see $[4,5])$

$$
F_{q}(x, t)=\sum_{n \geq 0}(-1)^{n} q^{\left(\begin{array}{c}
n \\
2
\end{array}\right)} \frac{e_{q}(x t) t^{2 n}}{[2 n]_{q} !}=\sum_{n \geq 0} H_{n, q}(x) \frac{t^{q}}{[n]_{q} !} .
$$

Recently, Waseem A. Khan and Divesh Srivastava (see [5, 12-14]) introduced the generalized $q$-Hermite-based Apostol-type polynomials ${ }_{H} \mathcal{F}_{n, q}^{(\alpha)}(x ; a, b ; \lambda ; \mu, \nu)$ by means of the generating function

$$
\left(\frac{2^{\mu} t^{\nu}}{\lambda e_{q}(t)+a^{b}}\right)^{\alpha} \sum_{n \geq 0}(-1)^{n} q^{\left(\begin{array}{c}
n \\
2
\end{array}\right)} \frac{e_{q}(x t) t^{2 n}}{[2 n]_{q} !}=\sum_{n \geq 0}{ }_{H} \mathcal{F}_{n, q}^{(\alpha)}(x ; a, b ; \lambda ; \mu, \nu) \frac{t^{q}}{[n]_{q} !}
$$

with $\alpha \in \mathbb{N}^{\star}, \lambda, a, b \in \mathbb{C}$ and $|t|<|\log (-\lambda)|$. Letting $x=0$ in the definition (6):

$$
{ }_{H} \mathcal{F}_{n, q}^{(\alpha)}(a, b ; \lambda ; \mu, \nu)={ }_{H} \mathcal{F}_{n, q}^{(\alpha)}(0 ; a, b ; \lambda ; \mu, \nu)
$$

are so called $q$-Hermite-based Apostol-type numbers of order $\alpha$ and generated by the function

$$
\left(\frac{2^{\mu} t^{\nu}}{\lambda e_{q}(t)+a^{b}}\right)^{\alpha} \sum_{n \geq 0}(-1)^{n} q^{\left(\begin{array}{c}
n \\
2
\end{array}\right)} \frac{t^{2 n}}{[2 n]_{q} !}=\sum_{n \geq 0}{ }_{H} \mathcal{F}_{n, q}^{(\alpha)}(a, b ; \lambda ; \mu, \nu) \frac{t^{q}}{[n]_{q} !} .
$$

Other interesting links about $q$-Hermite-based Apostol-type numbers, $(p ; q)$-analogue type of Frobenius Genocchi numbers and polynomials and $q$-analogue of Hermite poly-Bernoulli numbers and polynomials are illustrated in the works [6-11] of Waseem A. Khan et al.

\section{Explicit formula of generalized $q$-Hermite-based Apostol-type polynomials}

The generalized $q$-Apostol type polynomials $F_{n, q}^{(\alpha)}(x ; a, b ; \lambda)$ of order $\alpha \in \mathbb{N}^{\star}$ are defined by means of the generating function

$$
\left(\frac{2^{\mu} t^{\nu}}{\lambda e_{q}(t)+a^{b}}\right)^{\alpha} e_{q}(x t)=\sum_{n \geq 0} F_{n, q}^{(\alpha)}(x ; a, b ; \lambda) \frac{t^{n}}{[n]_{q} !}
$$

and the generalized $q$-Apostol type numbers $F_{n, q}^{(\alpha)}(a, b ; \lambda)=F_{n, q}^{(\alpha)}(0 ; a, b ; \lambda)$ are given by the generating function

$$
\left(\frac{2^{\mu} t^{\nu}}{\lambda e_{q}(t)+a^{b}}\right)^{\alpha}=\sum_{n \geq 0} F_{n, q}^{(\alpha)}(a, b ; \lambda) \frac{t^{n}}{[n]_{q} !} .
$$


Based on Cauchy product of series (see [1]); the $q$-analog Cauchy product of formal $q$-analog generating functions

$$
\sum_{n \geq 0} a_{n} \frac{t}{[n]_{q} !} \text { and } \sum_{n \geq 0} b_{n} \frac{t}{[n]_{q} !}
$$

is given by the following relation

$$
\left(\sum_{n \geq 0} a_{n} \frac{t}{[n]_{q} !}\right)\left(\sum_{n \geq 0} b n \frac{t}{[n]_{q} !}\right)=\sum_{n \geq 0} \sum_{k=0}^{n}\left(\begin{array}{l}
n \\
k
\end{array}\right)_{q} a_{k} b_{n-k} \frac{t^{n}}{[n]_{q} !} .
$$

Regarding the generating function of generalized $q$-Hermite-based Apostol-type polynomials; ${ }_{H} \mathcal{F}_{n, q}^{(\alpha)}(x ; a, b ; \lambda ; \mu, \nu)$ follows from $q$-analog Cauchy product of $\left(\frac{2^{\mu} t^{\nu}}{\lambda e_{q}(t)+a^{b}}\right)^{\alpha}$ and $F_{q}(x, t)$. By means of identity (10) we have

$$
{ }_{H} \mathcal{F}_{n, q}^{(\alpha)}(x ; a, b ; \lambda ; \mu, \nu)=\sum_{k=0}^{n}\left(\begin{array}{l}
n \\
k
\end{array}\right){ }_{q} F_{k, q}^{(\alpha)}(a, b ; \lambda) H_{n-k, q}(x) .
$$

To get explicit formula of ${ }_{H} \mathcal{F}_{n, q}^{(\alpha)}(x ; a, b ; \lambda ; \mu, \nu)$ we must compute the corresponding explicit formulae of numbers $F_{n, q}^{(\alpha)}(a, b ; \lambda)$ and polynomials $H_{n, q}(x)$.

\subsection{Explicit formula of $q$-Hermite polynomials}

$q$-Hermite polynomials follow from $q$-analog Cauchy product of

$$
e_{q}(x t) \text { and } F_{q}(t)=\sum_{n \geq 0}(-1) q^{\left(\begin{array}{c}
n \\
2
\end{array}\right)} \frac{t^{n}}{[n]_{q} !} .
$$

Explicitly we have the following theorem.

\section{Theorem 2.1.}

$$
H_{n, q}(x)=\sum_{k=0}^{\left\lfloor\frac{n}{2}\right\rfloor}(-1)^{k} q^{\left(\begin{array}{c}
k \\
2
\end{array}\right)}\left(\begin{array}{c}
n \\
2 k
\end{array}\right) x_{q} x^{n-2 k}
$$

Proof. First let the sequence $a_{n}$ be given by

$$
a_{n}=\frac{1}{2}\left(1+(-1)^{n}\right)(-1)^{\left\lfloor\frac{n}{2}\right\rfloor} q^{\left(\frac{\left\lfloor\frac{n}{2}\right\rfloor}{2}\right)} .
$$

Then

$$
F_{q}(t)=\sum_{n \geq 0} a_{n} \frac{t^{2 n}}{[2 n]_{q} !}
$$

and

$$
F_{q}(x, t)=\left(\sum_{n \geq 0} a_{n} \frac{t^{n}}{[n]_{q} !}\right)\left(\sum_{n \geq 0} \frac{x^{n} t^{n}}{[n]_{q} !}\right)
$$

Thus

$$
F_{q}(x, t)=\sum_{n \geq 0} \sum_{k=0}^{n}\left(\begin{array}{l}
n \\
k
\end{array}\right)_{q} a_{k} x^{n-k} \frac{t^{n}}{[n]_{q} !},
$$


but

$$
\sum_{k=0}^{n}\left(\begin{array}{l}
n \\
k
\end{array}\right)_{q} a_{k} x^{n-k}=\sum_{k=0}^{\left\lfloor\frac{n}{2}\right\rfloor}(-1)^{k} q^{\left(\begin{array}{c}
k \\
2
\end{array}\right)}\left(\begin{array}{c}
n \\
2 k
\end{array}\right)_{q} x^{n-2 k}
$$

and the result follows.

\section{$2.2 \alpha$-power $q$-analog generating function}

To compute the explicit formula of $\alpha$-power $q$-analog generating function; we must revisit some advanced studies in this area. Consider the formal generating function $f(t)=\sum_{n>0} a_{n} t^{n}$ with the coefficients $a_{n}$ are numbers or polynomials and the first term $a_{0} \neq 0$. Then $f^{\alpha}(t)$ is a generating function too, with hint of umbral calculus we noted in [3] that

$$
f^{\alpha}(t)=\sum_{n \geq 0} \sum_{a_{i_{1}}+\cdots+a_{i_{n}}=\alpha} a_{i_{1}} \ldots a_{i_{n}} t^{n} .
$$

In the general case $\alpha \in \mathbb{C}^{\star}$; an improvement of this result is given in our recent work [2], where

$$
f^{\alpha}(t)=a_{0}^{\alpha}+\sum_{n \geq 1} \sum_{k=1}^{n} \sum_{s_{n}(k)}\left(\begin{array}{l}
\alpha \\
k
\end{array}\right)\left(\begin{array}{c}
k \\
k_{1}, \ldots, k_{n}
\end{array}\right) a_{0}^{\alpha-k} a_{1}^{k_{1}} \ldots a_{n}^{k_{n}} t^{n},
$$

$s_{n}(k)$ is the set of all $\left(k_{1}, \ldots, k_{n}\right) \in \mathbb{N}^{n}$ satisfying conditions $k_{1}+\cdots+k_{n}=k$ and $k_{1}+2 k_{2}+\cdots+n k_{n}=n$. It is obvious to remark that $k_{j}=0$ for $j \geq n-k+1$ and $s_{n}(k)$ reduces to $(n-k+1)$-uplet $\left(k_{1}, \ldots, k_{n-k+1}\right)$. We conclude that

$$
f^{\alpha}(t)=a_{0}^{\alpha}+\sum_{n \geq 1} \sum_{k=1}^{n}(\alpha)_{k} a_{0}^{\alpha-k} B_{n, k}\left(1 ! a_{1}, \ldots,(n-k+1) ! a_{n-k+1}\right) \frac{t^{n}}{n !} .
$$

$B_{n, k}$ are exponential partial Bell polynomials given by the expression

$$
B_{n, k}\left(x_{1}, \ldots, x_{n-k+1}\right)=\frac{n !}{k !} \sum_{s_{n}(k)}\left(\begin{array}{c}
k \\
k_{1}, \ldots, k_{n-k+1}
\end{array}\right) \prod_{r=1}^{n-k+1}\left(\frac{x_{r}}{r !}\right)^{k_{r}}
$$

and defined by means of the generating function

$$
\frac{1}{k !}\left(\sum_{m \geq 1} x_{m} \frac{z^{m}}{m !}\right)=\sum_{n \geq k} B_{n, k}\left(x_{1}, \ldots, x_{n-k+1}\right) \frac{z^{n}}{n !} .
$$

Stirling numbers $S_{2}(n, k)$ obtained by the function

$$
\frac{1}{k !}\left(e^{t}-1\right)^{k}=\sum_{n \geq 0} S_{2}(n, k) \frac{t^{n}}{n !}
$$

are special case of $B_{n, k}$ and we have $B_{n, k}(1, \ldots, 1)=S_{2}(n, k)$. Consequently these polynomials admit the following formulation

$$
S_{2}(n, k)=\frac{1}{k !} \sum_{j=1}^{k}\left(\begin{array}{l}
k \\
j
\end{array}\right)(-1)^{k-j} j^{n} .
$$

According to exponential partial Bell polynomials, the explicit formula of $q$-analog generating function $f_{q}(t)=\sum_{n \geq 0} b_{n} \frac{t^{n}}{[n]_{q} !}$ is given by the following theorem. 


\section{Theorem 2.2.}

$$
f_{q}^{\alpha}(t)=b_{0}^{\alpha}+\sum_{n \geq 1} \sum_{k=1}^{n} \frac{(\alpha)_{k} b_{0}^{\alpha-k}[n]_{q} !}{n !} B_{n, k}\left(\frac{r ! b_{r}}{(q, q)_{r}}\right)(1-q)^{n} \frac{t^{n}}{[n]_{q} !},
$$

where

$$
B_{n, k}\left(\frac{r ! b_{r}}{(q, q)_{r}}\right)=B_{n, k}\left(\frac{1 ! b_{1}}{(q, q)_{1}}, \ldots, \frac{(n-k+1) ! b_{n-k+1}}{(q, q)_{n-k+1}}\right) .
$$

Proof. Let the sequence $a_{n}=\frac{b_{n}}{(n)_{q} !}$. Then $f_{q}(t)=\sum_{n \geq 0} a_{n} t^{n}$ and by means of the expression (15) we deduce that

$$
f_{q}^{\alpha}(t)=b_{0}^{\alpha}+\sum_{n \geq 1} \sum_{k=1}^{n} \frac{(\alpha)_{k} a_{0}^{\alpha-k}[n]_{q} !}{n !} B_{n, k}\left(\frac{1 ! b_{1}}{(1)_{q} !}, \ldots, \frac{(n-k+1) ! b_{n-k+1}}{(n-k+1)_{q} !}\right) \frac{t^{n}}{[n]_{q} !} .
$$

But

$$
B_{n, k}\left(\frac{1 ! b_{1}}{(1)_{q} !}, \ldots, \frac{(n-k+1) ! b_{n-k+1}}{(n-k+1)_{q} !}\right)=(1-q)^{n} B_{n, k}\left(\frac{1 ! b_{1}}{(q, q)_{1}}, \ldots, \frac{(n-k+1) ! b_{n-k+1}}{(q, q)_{n-k+1}}\right) .
$$

Then

$$
f^{\alpha}(t)=b_{0}^{\alpha}+\sum_{n \geq 1} \sum_{k=1}^{n} \frac{(\alpha)_{k} a_{0}^{\alpha-k}[n]_{q} !}{n !} B_{n, k}\left(\frac{r ! b_{r}}{(q, q)_{r}}\right)(1-q)^{n} \frac{t^{n}}{[n]_{q} !} .
$$

Let auxiliary sequence $c_{n}$ of numbers be defined by means of the generating function

$$
\left(\frac{1}{\lambda e_{q}(t)+a^{b}}\right)^{\alpha}=\sum_{n \geq 0} c_{n} \frac{t^{n}}{[n]_{q} !} .
$$

According to Theorem 2.2 it follows that $c_{n}$ is written in the form given by the following proposition.

Proposition 2.3. Let $\lambda+a^{b} \neq 0$. Then $c_{0}=\left(\lambda+a^{b}\right)^{-\alpha}$ and for $n \geq 1$ we have

$$
c_{n}=\sum_{k=1}^{n} \frac{(-\alpha)_{k}\left(\lambda+a^{b}\right)^{-\alpha-k}[n]_{q}}{n !} \lambda^{k}(1-q)^{n} B_{n, k}\left(\frac{r !}{(q, q)_{r}}\right) \text {. }
$$

Proof. The series expansion of $\lambda e_{q}(t)+a^{b}$ is

$$
\lambda e_{q}(t)+a^{b}=\lambda+a^{b}+\sum_{n \geq 1} \lambda \frac{t^{n}}{[n]_{q} !} .
$$

Then

$$
\left(\lambda e_{q}(t)+a^{b}\right)^{-\alpha}=\left(\lambda+a^{b}\right)^{-\alpha}+\sum_{n \geq 1} \sum_{k=1}^{n} \frac{(-\alpha)_{k}\left(\lambda+a^{b}\right)^{-\alpha-k}[n]_{q} !}{n !} \lambda^{k} B_{n, k}\left(\frac{r !}{(q, q)_{r}}\right)(1-q)^{n} \frac{t^{n}}{[n]_{q} !} .
$$

Furthermore $c_{0}=\left(\lambda+a^{b}\right)^{-\alpha}$ and for $n \geq 1$;

$$
c_{n}=\sum_{k=1}^{n} \frac{(-\alpha)_{k}\left(\lambda+a^{b}\right)^{-\alpha-k}[n]_{q} !}{n !} \lambda^{k}(1-q)^{n} B_{n, k}\left(\frac{r !}{(q, q)_{r}}\right) .
$$


Corollary 2.3.1. We have $F_{n, q}^{(\alpha)}(a, b ; \lambda)=0$ for $n<\nu \alpha, F_{\nu \alpha, q}^{(\alpha)}(a, b ; \lambda)=2^{\mu \alpha}\left(\lambda+a^{b}\right)^{-\alpha}[\nu \alpha]_{q}$ ! and for $n>\nu \alpha$ :

$$
F_{n, q}^{(\alpha)}(a, b ; \lambda)=2^{\mu \alpha}[n]_{q} ! \sum_{k=1}^{n-\nu \alpha} \frac{(-\alpha)_{k}\left(\lambda+a^{b}\right)^{-\alpha-k}}{(n-\nu \alpha) !}(1-q)^{n-\nu \alpha} \lambda^{k} B_{n-\nu \alpha, k}\left(\frac{r !}{(q, q)_{r}}\right) .
$$

Proof. We have

$$
\left(\frac{2^{\mu} t^{\nu}}{\lambda e_{q}(t)+a^{b}}\right)^{\alpha}=2^{\mu \alpha} t^{\nu \alpha}\left(\frac{1}{\lambda e_{q}(t)+a^{b}}\right)^{\alpha}
$$

Then,

$$
\left(\frac{2^{\mu} t^{\nu}}{\lambda e_{q}(t)+a^{b}}\right)^{\alpha}=2^{\mu \alpha} t^{\nu \alpha}\left(\left(\lambda+a^{b}\right)^{-\alpha}+\sum_{n \geq 1} c_{n} \frac{t^{n}}{[n]_{q} !}\right)
$$

Furthermore,

$$
\left(\frac{2^{\mu} t^{\nu}}{\lambda e_{q}(t)+a^{b}}\right)^{\alpha}=2^{\mu \alpha}\left(\lambda+a^{b}\right)^{-\alpha} t^{\nu \alpha}+2^{\mu \alpha} \sum_{n \geq \nu \alpha+1} c_{n-\nu \alpha} \frac{t^{n}}{[n-\nu \alpha]_{q} !} .
$$

Finally,

$$
\sum_{n \geq 0} F_{n, q}^{(\alpha)}(a, b ; \lambda) \frac{t^{n}}{[n]_{q} !}=2^{\mu \alpha}\left(\lambda+a^{b}\right)^{-\alpha}[\nu \alpha]_{q} ! \frac{t^{\nu \alpha}}{[\nu \alpha]_{q} !}+2^{\mu \alpha} \sum_{n \geq \nu \alpha} \frac{[n]_{q} ! c_{n-\nu \alpha}}{[n-\nu \alpha]_{q} !} \frac{t^{n}}{[n]_{q} !} .
$$

Then $F_{n, q}^{(\alpha)}(a, b ; \lambda)=0$ for $n<\nu \alpha, F_{\nu \alpha, q}^{(\alpha)}(a, b ; \lambda)=2^{\mu \alpha}\left(\lambda+a^{b}\right)^{-\alpha}[\nu \alpha]_{q}$ ! and for $n \geq \nu \alpha$ we have

$$
F_{n, q}^{(\alpha)}(a, b ; \lambda)=2^{\mu \alpha} \frac{[n]_{q} !}{[n-\nu \alpha]_{q} !} c_{n-\nu \alpha} .
$$

Substitute the value of $c_{n-\nu \alpha}$ to get the desired result.

We have already found the necessary tools for computing the explicit formula of $q$-Hermitebased Apostol-type polynomial.

Theorem 2.4. If $\lambda+a^{b} \neq 0$ we have ${ }_{H} \mathcal{F}_{n, q}^{(\alpha)}(x ; a, b ; \lambda ; \mu, \nu)=0$ for $n<\nu \alpha$ and for $n \geq \nu \alpha$ :

$$
\begin{aligned}
& { }_{H} \mathcal{F}_{n, q}^{(\alpha)}(x ; a, b ; \lambda ; \mu, \nu)=2^{\mu \alpha}\left(\lambda+a^{b}\right)^{-\alpha}[\nu \alpha]_{q} !\left(\begin{array}{c}
n \\
\nu \alpha
\end{array}\right) \sum_{q}^{\left\lfloor\frac{n-\nu \alpha}{2}\right\rfloor}(-1)^{l} q^{\left(\begin{array}{l}
l \\
2
\end{array}\right)}\left(\begin{array}{c}
n-\nu \alpha \\
2 l
\end{array}\right)_{q} x^{n-\nu \alpha-2 l} \\
& +2^{\mu \alpha} \sum_{1}\left(\begin{array}{l}
n \\
k
\end{array}\right)_{q}\left(\begin{array}{c}
n-k \\
2 l
\end{array}\right)_{q}[k]_{q} !(1-q)^{k-\nu \alpha}(-1)^{l} \lambda^{j}(-\alpha)_{j} \\
& \times q^{\left(\begin{array}{l}
l \\
2
\end{array}\right)} \frac{\left(\lambda+a^{b}\right)^{-\alpha-j}}{(k-\nu \alpha) !} B_{k-\nu \alpha, j}\left(\frac{r !}{(q, q)_{r}}\right) x^{n-k-2 l},
\end{aligned}
$$

where $\sum_{1}$ is the triple sum $\sum_{k=\nu \alpha}^{n} \sum_{j=1}^{k-\nu \alpha} \sum_{l=0}^{\left\lfloor\frac{n-k}{2}\right\rfloor}$.

Proof. Since

$$
{ }_{H} \mathcal{F}_{n, q}^{(\alpha)}(x ; a, b ; \lambda ; \mu, \nu)=\sum_{k=\nu \alpha}^{n}\left(\begin{array}{l}
n \\
k
\end{array}\right)_{q} F_{k, q}^{(\alpha)}(a, b ; \lambda) H_{n-k, q}(x)
$$


and

$$
H_{n-k, q}(x)=\sum_{l=0}^{\left\lfloor\frac{n-k}{2}\right\rfloor}(-1)^{l} q^{\left(\begin{array}{l}
l \\
2
\end{array}\right)}\left(\begin{array}{c}
n-k \\
2 l
\end{array}\right)_{q} x^{n-k-2 l}
$$

Then

$$
\begin{aligned}
{ }_{H} \mathcal{F}_{n, q}^{(\alpha)}(x ; a, b ; \lambda ; \mu, \nu)= & 2^{\mu \alpha}\left(\lambda+a^{b}\right)^{-\alpha}[\nu \alpha]_{q} !\left(\begin{array}{c}
n \\
\nu \alpha
\end{array}\right)_{q} H_{n-\nu \alpha, q}(x) \\
& +\sum_{k=\nu \alpha+1}^{n}\left(\begin{array}{l}
n \\
k
\end{array}\right) F_{q} F_{k, q}^{(\alpha)}(a, b ; \lambda) H_{n-k, q}(x)
\end{aligned}
$$

and the desired result follows.

Remark 2.5. In the case $\lambda+a^{b}=0$ and $\lambda \neq 0$; the result is totally different. We write

$$
\lambda e_{q}(t)+a^{b}=t \sum_{n \geq 0} \lambda \frac{t^{n}}{[n+1]_{q} !} .
$$

We consider $\nu \geq 1$, then we will have

$$
\left(\frac{2^{\mu} t^{\nu}}{\lambda e_{q}(t)+a^{b}}\right)^{\alpha}=\left(\frac{2^{\mu}}{\lambda}\right)^{\alpha} t^{\nu \alpha-\alpha}\left(\frac{1}{\sum_{n \geq 0} \frac{t^{n}}{[n+1]_{q} !}}\right)^{\alpha}
$$

But

$$
\left(\frac{1}{\sum_{n \geq 0} \frac{t^{n}}{[n+1]_{q} !}}\right)^{\alpha}=1+\sum_{n \geq 1} \sum_{k=1}^{n} \frac{(-\alpha)_{k}[n]_{q} !}{n !} B_{n, k}\left(\frac{r !}{[n+1]_{q}(q, q)_{r}}\right) \times(1-q)^{n} \frac{t^{n}}{[n]_{q} !} .
$$

Then

$$
\begin{aligned}
\left(\frac{2^{\mu} t^{\nu}}{\lambda e_{q}(t)+a^{b}}\right)^{\alpha}= & \left(\frac{2^{\mu}}{\lambda}\right)^{\alpha} t^{\nu \alpha-\alpha}+\left(\frac{2^{\mu}}{\lambda}\right)^{\alpha} \sum_{n \geq 1} \sum_{k=1}^{n} \frac{(-\alpha)_{k}[n]_{q} !}{n !} \\
& \times B_{n, k}\left(\frac{r !}{[n+1]_{q}(q, q)_{r}}\right)(1-q)^{n} \frac{t^{n+\nu \alpha-\alpha}}{[n]_{q} !}
\end{aligned}
$$

and

$$
\begin{aligned}
\left(\frac{2^{\mu} t^{\nu}}{\lambda e_{q}(t)+a^{b}}\right)^{\alpha}= & \left(\frac{2^{\mu}}{\lambda}\right)^{\alpha} t^{c}+\left(\frac{2^{\mu}}{\lambda}\right)^{\alpha} \sum_{n \geq c+1} \sum_{k=1}^{n-c} \frac{(-\alpha)_{k}}{(n-c) !}(1-q)^{n-c} \\
& \times B_{n-c, k}\left(\frac{r !}{[n-c+1]_{q}(q, q)_{r}}\right) t^{n},
\end{aligned}
$$

where $c=\nu \alpha-\alpha$. Let us write

$$
\left(\frac{2^{\mu} t^{\nu}}{\lambda e_{q}(t)+a^{b}}\right)^{\alpha}=\sum_{n \geq 0} d_{n} \frac{t^{n}}{[n]_{q} !}
$$

Then $d_{n}=0$ for $n<c, d_{c}=\left(\frac{2^{\mu}}{\lambda}\right)^{\alpha}[c]_{q}$ ! and for $n \geq c+1$ we have

$$
d_{n}=\left(\frac{2^{\mu}}{\lambda}\right)^{\alpha} \sum_{k=1}^{n-c} \frac{(-\alpha)_{k}[n]_{q} !}{(n-c) !} B_{n-c, k}\left(\frac{r !}{[n-c+1]_{q}(q, q)_{r}}\right) \times(1-q)^{n-c} .
$$


By means of the identity (11) we will have ${ }_{H} \mathcal{F}_{n, q}^{(\alpha)}(x ; a, b ; \lambda ; \mu, \nu)=\sum_{k=c}^{n}\left(\begin{array}{l}n \\ k\end{array}\right)_{q} d_{k} H_{n-k, q}(x)$.

Finally for $n \geq c$

$$
\begin{aligned}
& { }_{H} \mathcal{F}_{n, q}^{(\alpha)}(x ; a, b ; \lambda ; \mu, \nu)=\left(\frac{2^{\mu}}{\lambda}\right)^{\alpha}[c]_{q} ! \sum_{l=0}^{\left\lfloor\frac{n-c}{2}\right\rfloor}(-1)^{l} q^{\left(\begin{array}{l}
l \\
2
\end{array}\right)}\left(\begin{array}{c}
n-c \\
2 l
\end{array}\right)_{q} x^{n-c-2 l}+
\end{aligned}
$$

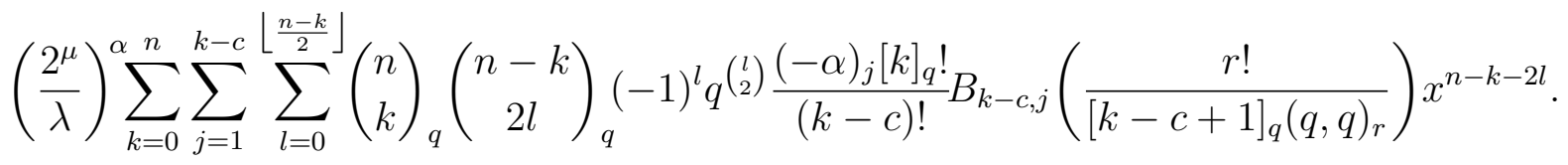

\section{Generalized $f_{q}$-Hermite-based Apostol-type polynomials}

Let $\alpha \neq 0$ be a complex number and $\beta$ real number. We consider the formal $q$-analog generating function $f_{q}(t)=\sum_{n \geq 0} b_{n} \frac{t^{n}}{[n]_{q} !}$ with the condition that $b_{0}$ is different from zero. A natural generalization of $q$-Hermite-based Apostol-type polynomials is given by the following definition

Definition 3.1. The $f_{q}$-Hermite-based Apostol-type polynomials ${ }_{H} \mathcal{F}_{n, f_{q}}^{(\alpha)}(x ; a, b ; \lambda ; \mu, \nu)$ are given by the generating function

$$
\beta t^{m} f_{q}^{\alpha}(t) e_{q}(x t) \sum_{n \geq 0}(-1)^{n} q^{\left(\begin{array}{c}
n \\
2
\end{array}\right)} \frac{t^{2 n}}{[2 n]_{q} !}=\sum_{n \geq 0} F_{n, f_{q}}^{(\alpha)}(x ; \beta ; c) \frac{t^{n}}{[n]_{q} !} .
$$

Thereafter the $f_{q}$-Hermite-based Apostol-type numbers $F_{n, f_{q}}^{(\alpha)}(\beta ; c)=F_{n, f_{q}}^{(\alpha)}(0 ; \beta ; c)$ are given by the generating function

$$
\beta t^{m} f_{q}^{\alpha}(t) \sum_{n \geq 0}(-1)^{n} q^{\left(\begin{array}{c}
n \\
2
\end{array}\right)} \frac{t^{2 n}}{[2 n]_{q} !}=\sum_{n \geq 0} F_{n, f_{q}}^{(\alpha)}(\beta ; c) \frac{t^{n}}{[n]_{q} !} .
$$

For $-\alpha \in \mathbb{N}^{\star}, \beta=2^{\mu \alpha}, m=\nu \alpha$ and $f_{q}(t)=\lambda e_{q}(t)+a^{b} ; F_{n, f_{q}}^{(\alpha)}(x ; \beta)={ }_{H} \mathcal{F}_{n, q}^{(\alpha)}(x ; a, b ; \lambda ; \mu, \nu)$. Polynomials $F_{n, f_{q}}^{(\alpha)}(x ; \beta ; c)$ follows from $q$-analog Cauchy product of generating functions

$$
e_{q}(x t) \sum_{n \geq 0}(-1)^{n} q^{\left(\begin{array}{c}
n \\
2
\end{array}\right)} \frac{t^{2 n}}{[2 n]_{q} !}=\sum_{n \geq 0} H_{n, q}(x) \frac{t^{n}}{[n]_{q} !}
$$

and

$$
\beta t^{m} f_{q}^{\alpha}(t)=\sum_{n \geq 0} b_{n} \frac{t^{n}}{[n]_{q} !} .
$$

The closed formula of polynomial $F_{n, f_{q}}^{(\alpha)}(x ; \beta ; c)$ is established in the following theorem.

Theorem 3.2.

$$
\begin{array}{r}
F_{n, f_{q}}^{(\alpha)}(x ; \beta ; c)=\beta b_{0}^{\alpha}\left(\begin{array}{c}
n \\
m
\end{array}\right)_{q}[m]_{q} ! \sum_{l=0}^{\left\lfloor\frac{n-m}{2}\right\rfloor}(-1)^{l} q^{\left(\begin{array}{l}
l \\
2
\end{array}\right)\left(\begin{array}{c}
n-m \\
2 l
\end{array}\right)_{q} x^{n-m-2 l}+} \\
\beta \sum_{2}\left(\begin{array}{l}
n \\
k
\end{array}\right)_{q}\left(\begin{array}{c}
n-k \\
2 l
\end{array}\right)_{q} \frac{(\alpha)_{j} b_{0}^{\alpha-j}[k]_{q} !}{(k-m) !}(1-q)^{k-m}(-1)^{l} q^{\left(\begin{array}{l}
l \\
2
\end{array}\right)} B_{k-m, j}\left(\frac{r ! b_{r}}{(q, q)_{r}}\right) x^{n-k-2 l},
\end{array}
$$

where $\sum_{2}$ is the triple sum $\sum_{r=m+1}^{n} \sum_{j=1}^{k-m} \sum_{l=0}^{\left\lfloor\frac{n-k}{2}\right\rfloor}$. 
Proof. Since

$$
f_{q}^{\alpha}(t)=b_{0}^{\alpha}+\sum_{n \geq 1} \sum_{k=1}^{n} \frac{(\alpha)_{k} b_{0}^{\alpha-k}}{n !} B_{n, k}\left(\frac{r ! b_{r}}{(q, q)_{r}}\right)(1-q)^{n} t^{n}
$$

then

$$
t^{m} f_{q}^{\alpha}(t)=b_{0}^{\alpha}[m]_{q} ! \frac{t^{m}}{[m]_{q} !}+\sum_{n \geq m+1} \sum_{k=1}^{n-m} \frac{(\alpha)_{k} b_{0}^{\alpha-k}[n]_{q} !}{(n-m) !} B_{n-m, k}\left(\frac{r ! b_{r}}{(q, q)_{r}}\right)(1-q)^{n-m} \frac{t^{n}}{[n]_{q} !} .
$$

Writing

$$
\beta t^{m} f_{q}^{\alpha}(t)=\sum_{n \geq 0} c_{n} \frac{t^{n}}{[n]_{q} !}
$$

then $c_{n}=0$ for $n<m, c_{m}=\beta b_{0}^{\alpha}[m]_{q}$ ! and for $n>m$ we have

$$
c_{n}=\beta \sum_{k=1}^{n-m} \frac{(\alpha)_{k} b_{0}^{\alpha-k}[n]_{q} !}{(n-m) !} B_{n-m, k}\left(\frac{r ! b_{r}}{(q, q)_{r}}\right)(1-q)^{n-m}
$$

Thereafter in means of $q$-analog Cauchy product of generating functions we have

$$
F_{n, f_{q}}^{(\alpha)}(x ; \beta ; c)=\sum_{r=m}^{n}\left(\begin{array}{l}
n \\
k
\end{array}\right)_{q} c_{k} H_{n-k}(x)
$$

and the desired result follows.

\section{References}

[1] Goubi, M. (2018). Successive derivatives of Fibonacci type polynomials of higher order in two variables, Filomat, 32, 5149-5159.

[2] Goubi, M. (2020). A new class of generalized polynomials associated with HermiteBernoulli polynomials, J. Appl. Math. \& Informatics, 38, 211-220.

[3] Goubi, M. (2019). On the Recursion Formula of Polynomials Generated by Rational Functions, Inter. Journ. Math. Analysis, 13, 29-38.

[4] Mahmudov, N. I. (2014). Difference equations of q-Appell polynomials, Appl. Math. Comput., 245, 539-543.

[5] Khan, W. A., \& Srivastava, D. (2020). A new class of $q$-Hermite-based Apostol-type polynomials and its applications, Notes on Number Theory and Discrete Mathematics, $26(1), 75-85$.

[6] Khan, W. A., Khan, I. A., \& Musharraf A. (2020). Degenerate Hermite poly-Bernoulli numbers and polynomials with $q$-parameter, Stud. Univ. Babes-Bolayi, Math., 65 (1), 3-15.

[7] Khan, W. A., \& Khan, I. A. (2020). A note on $(p, q)$-analogue type of Frobenius Genocchi numbers and polynomials. East Asian Mathematical Journal, 36 (1), 13-24. 
[8] Khan, W. A., \& Nisar, K. S. (2019). Notes on $q$-Hermite based unified Apostol type polynomials. Journal of Interdisciplinary Mathematics, 22 (7), 1185-1203.

[9] Khan, W. A., Khan, I. A., \& Musharraf, A. (2019). A note on $q$-analogue of Hermite-polyBernoulli numbers and polynomials. Mathematica Morvica, 23 (2), 1-16.

[10] Kang, J. Y., \& Khan, W. A. (2020). A new class of $q$-Hermite-based Apostol type Frobenius Genocchi polynomials. Communication of the Korean Mathematical Society, 35 (3), 759-771.

[11] Khan, W. A., Khan, I. A., Acikgoz, M., \& Duran, U. (2020). Multifarious results for $q$-Hermite based Frobenius type Eulerian polynomials. Notes on Number Theory and Discrete Mathematics, 26 (2), 127-141.

[12] Khan, W. A., \& Srivastava, D. (2019). A study of poly-Bernoulli polynomials associated with Hermite polynomials with q-parameter. Honam Mathematical J., 41 (4), 781-798.

[13] Khan, W. A., \& Srivastava, D. (2019). On the generalized Apostol-type Frobenius-Genocchi polynomials, Filomat, 33 (7), 1967-1977.

[14] Khan, W. A., \& Srivastava, D. (2021). Certain properties of Apostol-type Hermite-basedFrobenius-Genocchi polynomials, Kragujevac Journal of Mathematics, 45 (6), 859-872. 